\title{
The Jets in Micro-Quasars and Quasars: A Comparison
}

\author{
Ralph Spencer \\ Jodrell Bank Observatory, University of Manchester, Macclesfield, \\ Cheshire SK11 9DL,UK
}

\begin{abstract}
The discovery of relativistic jets in radio emitting $\mathrm{X}$-ray binaries in our own galaxy has led to the term 'microquasar' being coined for these objects. In this paper the properties of microquasars are compared with those of quasars and radio galaxies, with the aim of trying to see how the similarities and differences can inform us about the physical conditions and evolution of jets. GRS1915+105 and SS433 are also discussed in more detail.
\end{abstract}

\section{Introduction}

$\mathrm{X}$-ray binary stars display a wide range of interesting phenomena particularly in X-rays and perhaps offer the best laboratory for the study of the accretion process and the physics of extreme environments. The strong X-rays indicate the existence of a deep gravitational potential well and so imply the presence of a neutron star or black hole. Radio emission has been found in $\sim 50$ of the 280 known X-ray binaries listed in the catalogues of Liu et al. $(2000,2001)$ of high and low mass binaries (e.g. Fender 2003). Radio emission is a strong indicator of the presence of a radio jet. If the maximum brightness temperature (neglecting beaming effects) is limited by inverse Compton radiation to $10^{12} \mathrm{~K}$, then a diameter of 1 au for a source of $100 \mathrm{mJy}$ at $5 \mathrm{GHz}$ at a distance of 1 $\mathrm{kpc}$ is implied. The radio emission therefore extends beyond the likely diameter of an accretion disk around a compact object, and the variability suggests that the emitting region is ejected from the central engine. Indeed high resolution observations show that about half of the radio-emitting X-ray binaries (REXRB) have radio jets.

The first REXRB found to show apparent superluminal velocities of knots in its radio jets was GRO J1655-40 (Tingay et al. 1995). Since then a further 5 objects have been found to show features moving down the radio jet at apparent speeds >c (Fender 2003). This phenomenon, together with the presence of a jet has led to the objects being called 'Microquasars' by analogy to the more powerful (and $\sim 10^{6}$ times further away) quasars (Mirabel et al. 1992, Mirabel \& Rodríguez 1999). The fundamental energy source is the same i.e. they are powered by accretion onto a compact object. Perhaps all REXRBs should be labeled microquasars, if indeed they all have radio jets.

\section{Radio Properties}

Properties of microquasars such as the radio morphology, component proper motions and other derived parameters can be compared with those of extragalactic objects covering a wide range in luminosity from the relatively nearby Active Galactic Nuclei to the distant quasars (e.g. De Young 2002). Many images of 
the radio emission from extragalactic sources have been published, a convenient compendium is given by the Atlas of DRAGNs by Leahy, Bridle \& Strom (2000).

\subsection{Morphology}

The most obvious difference between REXRB and quasars is that there are no obvious extended lobes situated on either side of the REXRB for nearly all the known objects. The exceptions are the nebulae which surround SS433 (W50) (Dubner et al. 1998) and Circ X-1 (Haynes et al. 1986). These extended regions are likely to be the remains of the supernovae associated with the formation of the compact objects rather than formed by the action of the jets alone. However the 'ears' of W50 are probably formed by the precessing jets of SS433 moving out into the interstellar medium, and so perhaps can be thought of as the equivalent of lobes. Similarly the jet in Circ X-1 may be passing energy into its nebula (Fender et al. 1998), analogous to M87 where the jet appears to feed into an extended halo (Owen, Eilek \& Kassim, 2000).

Similarly there are no 'hotspots', where the jets may be interacting with the interstellar medium in a working surface. Compact IRAS sources have been found aligned with GRS1915+105 (Rodríguez \& Mirabel 1999), but these may be HII regions.

The morphology of REXRB jets is similar to that of the core-jet quasars like 3C345 and 3C273, etc., and also the low luminosity FRI type of radio source where the jets are brighter towards the nucleus of the object. The jet in BL Lac (Stirling et al. 2003) looks like the jets in Cyg X-1 and SS433. Indeed the precession found in BL Lac is an interesting result, suggesting a close analogy to SS433 and is worth investigating further. We should therefore think of microquasars as naked jet sources, though the energy flowing through the jet must be deposited somewhere in the interstellar medium.

\subsection{Proper Motions}

Measurements of the proper motion of components within microquasar jets lead to estimates of velocities ranging from $\sim 0.3$ to $0.98 \mathrm{c}$ or more. While the high velocity objects have Lorentz factors which are not too dissimilar to those in quasars, there is an outstandingly different effect. Several objects have been detected with two-sided ejections (e.g. GRO J1655-40, GRS 1915+105) even though their velocities are high, whereas jet-dominated quasars and BL Lac objects are exclusively one-sided. Doppler boosting causes this effect for quasars, which on the whole have been selected by their radio emission. Microquasars on the other hand have been selected by their X-ray emission, the bulk of which is not Doppler boosted.

\subsection{Derived Parameters}

Typical jet powers in microquasars are $\sim 10^{37} \mathrm{erg} \mathrm{sec}^{-1}$, compared to $10^{46} \mathrm{erg}$ $\mathrm{sec}^{-1}$ for quasars and FRII sources, and $10^{42} \mathrm{erg} \mathrm{sec}^{-1}$ for FRI objects (Spencer, de la Force \& Stirling 2001) We should therefore perhaps call REXRB NanoQuasars! The jet powers in microquasars are an appreciable fraction of the total accretion powers available $\left(10^{38}-10^{39} \mathrm{erg} \mathrm{sec}^{-1}\right)$; the fraction may approach unity if there there is a large contribution from protons. 
Jet synchrotron flux density has been shown to scale with black-hole mass ( $\propto \mathrm{M}^{1.4}$, Heinz \& Sunyaev 2003, Sunyaev 2003), implying that extragalactic jets are more radio loud and therefore suffer higher energy losses than microquasar jets, i.e. lose a higher fraction of their kinetic power in the form of radiation.

The length of jets in microquasars varies from $\sim 10$ au to $\sim 10^{6}$ au (Spencer et al. 2001). VLBI scale (10's au) "mini-jets" have been found in SS433 (Vermeulen et al. 1993) and GRS1915+105 (Dhawan, Mirabel \& Rodríguez 2000) as well as large scale jets extending over $10000 \mathrm{au}$. The mini-jet sources (e.g. Cyg $\mathrm{X}-1$, Stirling et al. 2001) have flat radio spectra and are rather similar to the flat spectrum core jet extragalactic sources in overall appearance. The different size scales however result in different conditions: a typical equipartition magnetic field of $\sim 100$ mgauss for microquasars means that the electron lifetime at $\mathrm{cm}$ wavelengths is much longer than the dynamical time and adiabatic rather than radiative losses dominate. There is no need for particle re-acceleration along the jets of microquasars in order to maintain the jet intensity at radio wavelengths. Jets in microquasars also propagate though a dense interstellar medium and so pressure in the jet needs to be higher than in quasars in order for a jet to propagate.

\section{Individual Microquasars}

GRS 1915+105 shows complicated X-ray behavior and is perhaps the best laboratory for studying accretion flows. Radio flaring has been associated with $\mathrm{X}$-ray outbursts, and flares are accompanied by the ejection of radio emitting components at velocities of $\sim 0.98 \mathrm{c}$ (Mirabel and Rodríguez 1994, Fender et al. 1999). The radio flares appear after a period of steady radio flux with a flat spectrum (the plateau state) when the X-ray emission changes from a steady low-hard state to a variable high flux soft state. Persistent mini-jets have been found when the source is in the plateau state, however this region is weaker after the strong flaring occurs.

It was possible to follow the motion of the ejected clouds in the Fender et al. (1999) observations by making images with MERLIN every 2 days. Extrapolating the positions of the components indicated an ejection date of around 1 day after the onset of the soft X-rays. Recent observations of a flaring event in July 2001 (McCormick 2003) confirm this behavior. It seems that a disk which is strong in soft X-rays is necessary for the ejection of radio clouds, but it is not required for the formation of the mini-jet.

It is interesting that the superluminal radio galaxy $3 \mathrm{C} 120$ also shows the ejection of radio clouds just after a restoration of high X-ray emission. Marscher et al. (2002) showed that a dip in X-rays occurred just before radio flaring. The presence of a newly formed and unstable disk seems to be necessary for the ejection of radio emitting components at relativistic velocities.

The presence of the low level compact jet in the low-hard state suggests that an underlying jet may exist at all times. The flaring could then be due to the development of a strong shock in the jet, rather than the ejection of a discrete cloud of relativistic electrons and magnetic field.

Shock in jet models (Marscher \& Gear 1985) have been used to describe high radio frequency outbursts in extragalactic core-jet objects like 3C273 (e.g. 
Türler, Courvoisier \& Paltani 2000) rather well. The outbursts in 3C273 are remarkably similar to those on GRS $1915+105$. Türler, Courvoisier \& Chaty (2003) have applied the model to the May 1997 outburst on GRS 1915+105. They predict a steep electron energy spectrum and rapid expansion of the jet (i.e a trumpet shape rather than a cone). Stevens et al. (2003) found that the variability of GRO J1655-40 can also be explained using the generalized shock model. However the expanding plasma cloud model has also been used successfully to describe the June 2001 outburst in GRS 1915+105 at 23-cm wavelength (Ishwara-Chandra, Yadav \& Pramesh Rao 2002). A detailed comparison of the two models applied to GRS $1915+105$ is clearly needed.

SS433 is famous for its precessing jets and has shown evidence for radio emission from a component extended roughly perpendicular to the jet and centered close to the core (Paragi et al. 1999, Blundell et al. 2001). Regions of extended emission also exist in some DRAGNS where the 'back-flow' from the advancing lobes interact. To investigate this further we have undertaken 2dimensional hydrodynamic modeling (Smponias 2003) where multiple ejections of radiating knots takes place (as seen in MERLIN and VLBI observations of the SS433 jets). It appears that the multiple bow shocks interact and heat and compress gas in the central region. The effect is more dominant when a wind is present.

\section{Conclusions}

Micro-quasar jets are more similar to those in FRI radio galaxies and BLLacs rather than the more luminous FRIIs. Jet formation and ejection are strongly associated with changes in X-ray state for microquasars, and as recent results on 3C120 suggest perhaps for extragalactic sources also. Shock in jet models may work for both, further progress requires frequent multi-wavelength observations. Studies of jets in both microquasars and quasars complement each other and indeed lead to further understanding of relativistic jets.

\section{References}

Blundell, Mioduszewski, A., Muxlow, T., Podriadlovski, P., \& Rupen, M. 2001, ApJ, $562, \mathrm{~L} 79$

Dhawan, V., Mirabel, I. F., \& Rodríguez, L. F. 2000, ApJ, 543, 373

De Young, D. S., 2002, The Physics of Extragalactic Radio Sources, (Chicago: University of Chicago Press)

Dubner, G. M., Holdaway, M., Goss, W. M., \& Mirabel, I. F. 1998, AJ, 116, 1842

Fender, R. P., Spencer, R., Tzioumis, T., Wu, K., et al. 1998, ApJ, 506, L121

Fender, R. P., Garrington, S. T., MacKay, D. J., Muxlow, T. W. B., Pooley, G. G., Spencer, R. E., Stirling, A. M., \& Waltman. E. B. 1999, MNRAS, 304, 865

Fender, R. P. 2003, in Compact X-ray Sources, ed W. Lewin \& M. van der Klis (Cambridge: CUP) in press

Haynes, R. F., Komesaroff, M. M., Little, A. G., Jauncey, D. L., Caswell, J. L., Milne, D. K., Kesteven, M. J., Wellington, K. L., \& Preston, R. A. 1986, Nature, 324, 233

Heinz, S., \& Sunyaev, R. A. 2003, MNRAS, 343, L59 
Ishwara-Chandra, C.H., Yadav, J. S., \& Pramesh Rao, A., 2002 A\&A, 388, L33

Leahy, J. P., Bridle, A. H., \& Strom R. G. 2000, http://www.jb.man.ac.uk/atlas

Liu, Q. Z., van Paradijs, J., van den Heuvel, E. P. J. 2000, A\&AS, 147, 25

Liu, Q. Z., van Paradijs, J., van den Heuvel, E. P. J. 2001, A\&A, 368, 1021

Marscher, A. P., \& Gear W. K. 1985, ApJ, 298, 114

Marscher, A. P., Jorstad, S. G., Gómez, J-L., Aller, M. F., Terasranto, H., Lister, M. L., \& Stirling, A. M. 2002, Nature, 417, 625

McCormick, D. G. 2003, PhD Thesis, University of Manchester

Mirabel, I. F., Rodríguez, L. F., Cordier, B., Lebrun, P. J. 1992, Nature, 358, 215

Mirabel, I. F., \& Rodríguez, I. F. 1994, Nature, 371, 46

Mirabel, I. F., \& Rodríguez, L. F., 1999, ARA\&A, 37, 409

Owen, F. N., Eilek, J. A., \& Kassim, N. E. 2000, ApJ, 543, 611

Paragi, Z., Vermeulen, R., Fejes, I., Schilizzi, R., Spencer, R., Stirling, A. 1999, A\&A, 348,910

Rodríguez, L. F., \& Mirabel, I. F. 1999, A\&A, 340, L47

Smponias, T. 2003, PhD Thesis, University of Manchester

Spencer, R. E., de la Force, C., \& Stirling, A. 2001, in IAU Symp. 205, Galaxies and their Constituents at the Highest Angular Resolution, ed R. T. Schilizzi, (San Francisco: ASP), 264

Stevens, J., Hannikainen, D., Wu, K., Hunstead, R., \& McKay, D. 2003, MNRAS, 342, 623

Stirling, A. M., Spencer, R. E., de la Force, C. J., Garrett, M. A., Fender, R. P., \& Ogley, R. N. 2001, MNRAS, 327, 1273

Stirling, A. M. et al. (12 authors) 2003, MNRAS, 341, 405

Sunyaev, R. A. 2003, these proceedings

Tingay, S. J., Jauncey, D. L., Reynolds, J. E., Meier, D. L., et al. 1995, Nature, 374, 141

Türler, M., Courvoisier, T. J-L., \& Paltani, S. 2000, A\&A, 361, 850

Türler, M., Courvoisier, T. J-L., \& Chaty, S. 2003, in ASP Conf. Ser. High Energy Blazar Astronomy, (San Francisco: ASP), vol. 299, 239

Vermeulen, R. C., Schilizzi, R. T., Spencer, R. E., Romney, J. D., \& Fejes, I., 1993, A\&A, 270, 177 